\title{
Prevalence of Musculoskeletal Disorders and Its Association with Mental Health and Quality of Life among Working Physiotherapists
}

\author{
Sai Iyer ${ }^{1}$, Labdhi Shah², Chitra Sharma ${ }^{3}$ \\ ${ }^{1}$ M.P.T. (Musculoskeletal Science), ${ }^{2}$ Intern Student, ${ }^{3}$ Intern Student, \\ Neotech Institute of Physiotherapy, Shri Govind Guru University, Vadodara, India \\ Corresponding Author: Sai Iyer
}

DOI: https://doi.org/10.52403/ijshr.20220124

\begin{abstract}
Background: Musculoskeletal disorders (MSDs) are defined as "regional impairments describing as inflammation and degenerative diseases and disorders of the muscles, tendons, nerves, joints, and fascia. When the surrounding work environment, work events (work itself) and the performance of work contribute in causing such kind of disorders over a time period, then they are called work-related musculoskeletal disorders (WRMSDs). It results into range of symptoms like pain, ache, disability, discomfort, fatigue, mental stress and thus resulting into loss of work time, work restriction of task, transfer to another job, sickness absenteeism and reducing overall quality of life. Workplace stress occurs in the workplace or work related organization, and is defined as "a harmful mental and physical reaction coming from discordance between employee's ability, resources, and willingness and work requirement. Also stress coming from pain, discomfort can also alter the mental status of the person and can quality of life (QoL) of employees.
\end{abstract}

Purpose of the study: To determine the Prevalence of WRMSD among working physiotherapist and the objective was to find out the association of WRMSD with mental health and quality of life.

Methodology: In this study a google form was created using 3 questionnaires (Nordic Musculoskeletal questionnaire for determining the musculoskeletal disorder, Quality of life was assessed through WHO-BREF. Mental health was assessed by General health questionnaire (GH-12).

Results: The prevalence of work-related musculoskeletal disorders among the Physiotherapists is $82 \%$. It is also concluded that the low back and neck are the most common sites susceptible to injury followed by upper back and thighs. There was a significant association of Work related musculoskeletal disorders with quality of life only.

Conclusions: Work-related musculoskeletal disorders are an important health risk within the physiotherapy profession. Therefore, it is recommended that education programs on prevention and coping strategies for musculoskeletal symptoms in Physiotherapists should be made mandatory in order to decrease the risk of WRMSDs which will ultimately improve the quality of life.

Keywords: [Work-Related Musculoskeletal disorders, Pain, quality of Life, Mental health, Physiotherapists, Physiotherapy]

\section{INTRODUCTION}

According to the Protocol of 2002 to the Occupational Safety and Health Convention, 1981, the term "occupational disease" covers any disease contracted as a result of an exposure to risk factors arising from work activity. Work related musculoskeletal disorders (WRMSD) have been an extensively explored focus for many decades and are the main cause of occupational injury in developed and developing countries. ${ }^{1}$ 
Musculoskeletal disorders (MSDs) are defined as "regional impairments describing as inflammation and degenerative diseases and disorders of the muscles, tendons, nerves, joints, and fascia. When the surrounding work environment, work events (work itself) and the performance of work contribute in causing such kind of disorders over a time period, then they are called work-related musculoskeletal disorders (WRMSDs) ${ }^{2}$. WRMSD results into range of symptoms like pain (both acute and chronic), ache, disability and discomfort in bodily regions and other based on the structure of the body affected. This results into loss of work time, work restriction of task, transfer to another job, sickness absenteeism and reducing overall quality of life $^{2}$

WMSDs may progress in stages from mild to serve.

The symptoms like pain, aching tiredness will be the same in all stages, but the severity will be different. During the early or mild stage, the symptoms occur during the work shift but disappear at night and during days off work. No reduction of work performance. In the Intermediate or moderate stage, the symptoms occur early in the work shift and persist at night. Reduced capacity for repetitive work. In late stage symptoms along with weakness persist at rest. Inability to sleep and to perform light duties. $^{2}$

$$
\begin{aligned}
& \text { Work related musculoskeletal } \\
& \text { disorders are common among } \\
& \text { Physiotherapists because of the nature of the } \\
& \text { profession that is repetitive, labour intensive } \\
& \text { (transferring dependent patient, assisting } \\
& \text { with mat activities), involving direct contact } \\
& \text { with the patients, working in an awkward } \\
& \text { /static position for a long time, high force } \\
& \text { levels and lack of rest time or recovery } \\
& \text { period which may be painful during work or } \\
& \text { at rest (WHO, 1985). These work patterns } \\
& \text { will put concentration of force on various } \\
& \text { parts of the body like neck, low back, } \\
& \text { shoulder, elbow, wrist, knee, hips, ankle and } \\
& \text { may result into both acute and chronic } \\
& \text { WRMSDs. }
\end{aligned}
$$

Work-related musculoskeletal disorders cause substantial economic losses to individuals as well as to the community. According to Global Burden of Diseases (GBD), injuries, and risk factors study 2017, between 2007 and 2017, among the three causes which are observed to have resulted in a further increase in the number of all-age years lived with disability (YLDs), low back pain (LBP) is attributable to a further increase of $17.5^{3}$

Workplace stress occurs in the workplace or work related organization, and is defined as "a harmful mental and physical reaction coming from discordance between employee's ability, resources, and willingness and work requirement". A Literature reported that high-level workplace stress harms the body and mind and consequently results in laziness, leaves of absence, job transfers, and exhaustion. Also stress coming from pain, discomfort can also alter the mental status of the person and can quality of life (QoL) of employees ${ }^{4}$.

The assessment of QoL in patients with chronic diseases such as MSDs became one of the public health goals, since this strategy allows the improvement of actions for the prevention and treatment of disease, from their physical, mental and social aspects $^{4}$.

Due to constant disability (based on stages), pain, discomfort, and by the burden of the disease and its impact on personal life, musculoskeletal disorders might result into alteration in mental health and reduced quality of life. Many studies have determined the prevalence of WRMSD among various occupations and in physiotherapy profession also. But from many studies it has been seen that pain, discomfort will lead to altered mental status and thus resulting in alterations in quality of life. Till the best of my knowledge no studied have did any association of prevalence with mental health and quality of life.

So this study will focus on the prevalence of WRMSD and its association with mental health and quality of life among 
working physiotherapist. So the aim of the study was to determine the prevalence of WRMSD among working Physiotherapist. The objective was to determine the association of WRMSD with mental health and quality of life among working Physiotherapists.

\section{MATERIALS \& METHODS}

Study Design: Cross-sectional study design Sample Size: 81, calculated by Sample size calculator for Cross sectional study

Sampling Method: Convenient Sampling

Settings: Physiotherapist working as clinical or academics in Vadodara, Anand and Bharuch

\section{Inclusion Criteria}

- Working physiotherapist in both clinical and academics.

- Age group (24-50 years)

- Both the genders.

Exclusion Criteria

- Not willing to participate.
Procedure: After getting the approval from ethical committee of concerned institution, a google form was made consisting of all 3 questionnaires and the demographic characteristics. WRMSD was measured by Nordic Musculoskeletal questionnaire. Quality of life was assessed through WHO-BREF. Mental health was assessed by General health questionnaire (GH-12). All the questionnaires were standard, reliable and valid to assess the functions ${ }^{(5,6,7)}$. The link of the form was send to many Physiotherapists across the city through WhatsApp. The description of the study and the consent was kept in the form.

\section{Statistical Analysis}

Data analysis was done using SPSS version 23.0. For estimation of Prevalence of WRMSD, descriptive analysis of the data was done. For comparison of WRMSD, with mental health status and quality of life, Kruskal Wallis test was done based on skewed distribution of data. $\mathrm{p}<0.05$

\section{RESULT}

Table 1: Demographic and Sociodemographic characteristics of Participants

\begin{tabular}{|c|c|c|c|}
\hline \multicolumn{2}{|l|}{ Variables } & $\mathbf{N}(\%)$ & Mean \pm SD \\
\hline \multirow[t]{2}{*}{ Gender } & Males & $34(42)$ & \\
\hline & Females & $47(58)$ & \\
\hline \multirow[t]{3}{*}{ Age } & $22-30$ & $49(60)$ & $24.10 \pm 2.45$ \\
\hline & $31-40$ & $27(34)$ & $35.30 \pm 2.76$ \\
\hline & $41-50$ & $5(6)$ & $46 \pm 3.3$ \\
\hline \multirow[t]{4}{*}{ BMI } & $<18.5$ & $6(8)$ & \\
\hline & $18.5-24.9$ & $27(33)$ & \\
\hline & $25-29.9$ & $30(37)$ & \\
\hline & $>30$ & $18(22)$ & \\
\hline \multirow[t]{3}{*}{ Occupation } & Academic & $11(14)$ & \\
\hline & Clinical & $30(37)$ & \\
\hline & Both & $40(49)$ & \\
\hline Experience (in years) & & & $5.2 \pm 5.1$ \\
\hline Total working hours in a day & & & $7.16 \pm 1.57$ \\
\hline \multirow{8}{*}{ What do you currently practice? } & Orthopaedic Rehabilitation & $24(28)$ & \\
\hline & General Physical Therapy & $27(33)$ & \\
\hline & Neurological Rehabilitation & $16(18)$ & \\
\hline & Cardiopulmonary Rehabilitation & $6(7)$ & \\
\hline & Sports Physiotherapy & $1(1.2)$ & \\
\hline & Paediatrics & $1(1.2)$ & \\
\hline & Women's Health & $4(4.9)$ & \\
\hline & Geriatrics & $2(2.4)$ & \\
\hline Hours of contact with patients per day & & & $3.6 \pm 1.89$ \\
\hline Hours of teaching per day & & & $2.69 \pm 1.03$ \\
\hline \multirow[t]{2}{*}{ Mode of teaching } & Sitting & $45(88)$ & \\
\hline & Standing & $6(12)$ & \\
\hline Hours of sitting & & & $3.61 \pm 1.42$ \\
\hline Duration of problem & & & $2.67 \pm 2.39$ \\
\hline Any sick leave? & & $20(30)$ & \\
\hline The duration of symptoms in a day & & & $2.54 \pm 1.2$ \\
\hline Has the symptoms altered your way of working? & & $25(37)$ & \\
\hline Pain VAS & & & $4.55 \pm 2.5$ \\
\hline
\end{tabular}

(N= No of Participants, \% Frequency, SD- Standard Deviation, VAS- Visual analog Scale) 
Sai Iyer et.al. Prevalence of musculoskeletal disorders and its association with mental health and quality of life among working physiotherapists

A total 81 participants responded to the form containing (questionnaires). The demographic characteristics of participants are shown in Table 1.

Table 2: Mechanism of Injury of Participants

\begin{tabular}{|c|l|c|}
\hline Sr. No & Mechanism of Injury & Frequency (percentage) \\
\hline $\mathbf{1}$ & Applying modalities & $4(4.9)$ \\
\hline $\mathbf{2}$ & Transferring a patient & $5(6.1)$ \\
\hline $\mathbf{3}$ & Performing repetitive tasks & $5(6.1)$ \\
\hline $\mathbf{4}$ & Working overtime & $4(4.9)$ \\
\hline $\mathbf{5}$ & Performing manual therapy technique & $12(14.8)$ \\
\hline $\mathbf{6}$ & Maintaining a static position for a prolonged period of time & $17(20.9)$ \\
\hline $\mathbf{7}$ & Working in an awkward position & $20(24.6)$ \\
\hline
\end{tabular}

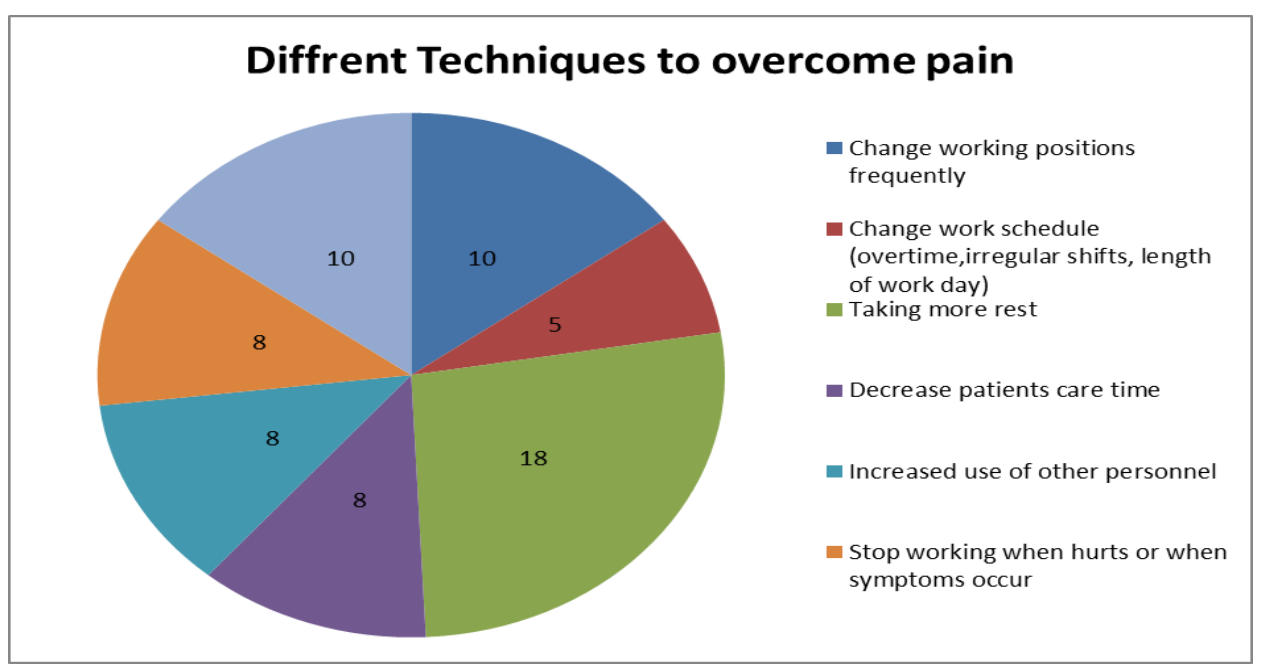

Graph 1: Different techniques adopted by Physiotherapist to overcome their pain Here the figure indicates Number of participants

\section{Prevalence of Work Related}

\section{Musculoskeletal disorder}

- Out of 81 responses from various physiotherapists working in various sectors, it was found that 67 among them had musculoskeletal disorder, and remaining has no any problem.

- So the prevalence of work related musculoskeletal disorder is $82.71 \%$.
- $18 \%$ therapists had no disorders.

- In the present study females were more affected than males. Out of a total of 47 females, 35 females has musculoskeletal disorders with a prevalence of $52 \%$ and out 34 males 32 were affected with musculoskeletal disorders with a prevalence of $48 \%$.

Table 3: Prevalence of WRMSD

\begin{tabular}{|c|c|c|c|}
\hline \multicolumn{4}{|c|}{ Prevalence of WRMSD according to the age groups } \\
\hline Sr. No & Age- Group & Frequency $(\mathbf{N})$ & Percentage $(\%)$ \\
\hline 1 & $22-30(49)$ & 35 & 71 \\
\hline 2 & $31-40(27)$ & 27 & 100 \\
\hline 3 & $41-50(05)$ & 5 & 100 \\
\hline \multicolumn{4}{|c|}{ Prevalence of WRMSD according to BMI } \\
\hline Sr. No & BMI & Frequency $(\mathbf{N})$ & Percentage $(\%)$ \\
\hline 4 & $<18$ & 4 & 66 \\
\hline 5 & $18-24.9 \quad(27)$ & 19 & 70 \\
\hline 6 & $25-30 \quad(30)$ & 28 & 93 \\
\hline 7 & $(18)$ & 16 & 88 \\
\hline \multicolumn{4}{|c|}{ Prevalence of WRMSD according to the Practicing Area } \\
\hline Sr. No & Area of Practice & Frequency $(\mathbf{N})$ & Percentage $(\%)$ \\
\hline 8 & Orthopedic $\quad$ Rehabilitation $\quad$ (24) & 23 & 95 \\
\hline 9 & General Physical Therapy $\quad$ (27) & 22 & 81 \\
\hline 10 & Cardiopulmonary Rehabilitation (6) & 03 & 50 \\
\hline 11 & Neurological Rehabilitation & 13 & 81 \\
\hline 12 & Sports & 01 & 100 \\
\hline 13 & Pediatrics (1) & 1 & 100 \\
\hline 14 & Women's Health (4) & 3 & 75 \\
\hline 15 & Geriatrics & 1 & 100 \\
\hline
\end{tabular}


Sai Iyer et.al. Prevalence of musculoskeletal disorders and its association with mental health and quality of life among working physiotherapists

\begin{tabular}{|c|c|c|c|}
\hline \multicolumn{4}{|c|}{ Table no 3: continued.... } \\
\hline \multicolumn{4}{|c|}{ Prevalence of WRMSD according to the occupation } \\
\hline Sr. No & Occupation & Frequency $(\mathbf{N})$ & Percentage (\%) \\
\hline 16 & Clinical (11) & 9 & 81 \\
\hline 17 & Academics (30) & 19 & 63 \\
\hline 18 & Both & 39 & 97 \\
\hline \multicolumn{4}{|c|}{ Prevalence of WRMSD according to the to the area affected } \\
\hline Sr. No & Area of the body Affected & Frequency $(\mathbf{N})$ & Percentage $(\%)$ \\
\hline 19 & Neck & 30 & 45 \\
\hline 20 & Upper back & 15 & 22 \\
\hline 21 & Lower back & 20 & 30 \\
\hline 22 & Thigh (Posterior aspect) & 2 & 3 \\
\hline \multicolumn{4}{|c|}{ Prevalence of WRMSD according to the Experience of Physiotherapists } \\
\hline Sr. No & Experience of Physiotherapists (years) & Frequency $(\mathbf{N})$ & Percentage $(\%)$ \\
\hline 23 & (36) & 35 & 97 \\
\hline 24 & $6-10$ & 17 & 68 \\
\hline 25 & $>10$ & 15 & 75 \\
\hline \multicolumn{4}{|c|}{ Prevalence of WRMSD based on Total working Hours of Physiotherapists } \\
\hline Sr. No & Total working Hours of Physiotherapists & Frequency $(\mathbf{N})$ & Percentage $(\%)$ \\
\hline 26 & (26) & 17 & 65 \\
\hline 27 & (55) & 50 & 90 \\
\hline
\end{tabular}

\begin{tabular}{|c|c|c|c|}
\hline \multicolumn{4}{|c|}{ Prevalence of WRMSD Based on Total Hours of Sitting by Physiotherapists } \\
\hline Sr. No & Total working Hours of Physiotherapists & Frequency $(\mathbf{N})$ & Percentage (\%) \\
\hline 1 & $(20)$ & 15 & 75 \\
\hline 2 & (50) & 45 & 90 \\
\hline 3. & (11) & 07 & 63 \\
\hline \multicolumn{4}{|c|}{$\begin{array}{l}\text { Prevalence of WRMSD Based on Total Hours in contact with patients by } \\
\text { Physiotherapists }\end{array}$} \\
\hline Sr. No & Total working Hours of Physiotherapists & Frequency $(\mathbf{N})$ & Percentage $(\%)$ \\
\hline 1 & $(50)$ & 30 & 60 \\
\hline 2 & (26) & 21 & 80 \\
\hline 3. & (5) & 5 & 100 \\
\hline \multicolumn{4}{|c|}{ Prevalence of WRMSD Based on Total Hours of teaching by Physiotherapists } \\
\hline Sr. No & Total working Hours of Physiotherapists & Frequency (N) & Percentage (\%) \\
\hline 1 & $(50)$ & 39 & 78 \\
\hline 2 & (31) & 14 & 45 \\
\hline \multicolumn{4}{|c|}{ Prevalence of WRMSD Based on Mode of teaching by Physiotherapists } \\
\hline Sr. No & Total working Hours of Physiotherapists & Frequency $(\mathbf{N})$ & Percentage $(\%)$ \\
\hline 1 & Sitting & 25 & 83 \\
\hline 2 & Standing (35) & 20 & 57 \\
\hline 3. & Both & 6 & 37 \\
\hline
\end{tabular}

(Figures next to the variable indicates frequency of participants within that particular variable).

Table 4: Scores of the 4 QOL domains, overall QOL

\begin{tabular}{|c|l|c|}
\hline Sr. No & Domains of WHO-BREF & Mean/SD \\
\hline 1 & Physical Domain & $51 \pm 5.6$ \\
\hline 2 & Psychological & $58 \pm 10.47$ \\
\hline 3. & Social Relationships & $83 \pm 15.95$ \\
\hline 4. & Environmental & $45 \pm 5.81$ \\
\hline
\end{tabular}

The second objective was to determine if there is any association of WRMSD on Mental health and quality of life.

Table 5: Analysis of Mental health status

\begin{tabular}{c|c|c}
-12 & Mean/SD & $17.87 \pm 6.90$ \\
(General Health care questionnaire-12)
\end{tabular}

Table 6: Comparison of WRMSD and WHO- BREF (Quality of life) Hypothesis Test Summary

\begin{tabular}{|l|l|l|lc|c|}
\hline Sr.No & \multicolumn{1}{|c|}{ Null Hypothesis } & \multicolumn{1}{c|}{ Test } & Sig. & Decision \\
\hline 1 & $\begin{array}{l}\text { The distribution of WHO-BREF is the } \\
\text { same across the categories of WRMSD }\end{array}$ & $\begin{array}{l}\text { Independent- Samples } \\
\text { Kruskal- Wallis Test }\end{array}$ & 0.040 & $\begin{array}{l}\text { Reject } \\
\text { Hypothesis. }\end{array}$ \\
\hline
\end{tabular}

*The test shows significant difference across the samples.

Table 7: Comparison of WRMSD and GH-12 (Mental Health Status) Hypothesis Test Summary

\begin{tabular}{|l|l|l|c|c|}
\hline Sr.No & \multicolumn{1}{|c|}{ Null Hypothesis } & \multicolumn{1}{c|}{ Test } & \multicolumn{1}{|c|}{ Sig. } & \multicolumn{1}{|c|}{ Decision } \\
\hline 1 & $\begin{array}{l}\text { The distribution of GH-12 is the same across the categories of } \\
\text { WRMSD }\end{array}$ & $\begin{array}{l}\text { Independent- } \\
\text { Samples } \\
\text { Kruskal- Wallis Test }\end{array}$ & $\begin{array}{l}0.638 \\
\text { the } \\
\text { Hypothesis. }\end{array}$ \\
\hline
\end{tabular}

(The significance level is 0.05 )

*The test shows no significant difference across the samples. 
The results showed that there is an association of WRMSD on quality of life but not on mental health among working Physiotherapists.

\section{DISCUSSION}

In this study a total of 81 responses came. So out of 81 responses, 67 Physiotherapists had work related musculoskeletal disorder. 14 therapists had no complains.

The prevalence rate was $82 \%$ among Physiotherapists. A study done by Obembe et al. (2008) and West et al. (2004) highlighted that the most common risk factors could be, dealing with an excessive number of patients in one day; continuing to work while injured or hurt; lifting or transferring dependent patients, improper work scheduling, working in a constant posture for a long period of time, not following proper ergonomics, performing manual therapy techniques in an awkward posture; and lack of recovery period of time

It was seen by the data that the prevalence rate among males and females is $48 \%$ and $52 \%$ respectively. But by carefully analyzing the data one might see that out of total 81 samples, 34 were males and out of that 34,32 were affected, and out of a total of 47 females, 35 were affected. So the affection was more in males compared to females. The possible reason could be higher job risk factors with "major problem" as compared to females. These include dealing with an excessive number of patients in one day; lifting or transferring dependent patients; continuing to work while injured or hurt; not enough rest breaks or pauses during the workday; performing the same task over and over: bending or twisting your back in an awkward way by the males. But other literature had another view, that females are more affected than males in case of WRMSD. The potential factor identified was their less height and more body weight which put them at a disadvantage during patients' treatment and transfer. ${ }^{(8,9)}$.
In the present study younger age groups had the most prevalence than the middle and older ones, even though there were less no of participants in older group. The possible explanation for this would be the younger therapists could be working in physically demanding areas and with less experience and skills early in their careers. Bork et al. have suggested that the ageprevalence trend, lower in older PTs, resulted from PTs moving out of patient care as they gained experience and into administrative positions that are less physically demanding. Also with gain in experience, the therapists might follow much ergonomic or alternative methods or coping strategies to avoid pain and discomfort ${ }^{10}$.

In the presence study the prevalence of WRMD was more in overweight group than in normal and in obese group. The possible reason which could explain this is overweight could factor to contribute in increasing of physiological and mechanical load on tissues. Relative disk pressure is being experienced during sitting with various inclinations of the back support. Intra-discal pressure of the nucleus pulpous acts as a load transducer and indicates the magnitude of axial loading on the spinal column and the increased pressure indicates a greater muscular effort in maintaining the posture and hence a larger stress on spinal column and could also result into psychological stress ${ }^{11}$.

Overweight yields a decreased postural stability and potentially negative impact on control of upper limb movements but its effect on control of balance imposes constraints on goal-directed movements. From a clinical perspective, obese individuals might be less efficient and more at risk of injuries than normal individuals in a large number of work tasks and daily activities especially requiring upper limb movements performed from an upright position. Overweight has got an impact on occupational-psychosocial stress. Because of repetitive movements of upper limbs, 
completion of a certain task in a stipulated time period, competition with fellow colleagues put the overweight and obese workers in a major occupational stress. The reason for individuals with normal BMI with getting WRMSD would be not following proper ergonomics, not maintaining proper position while treating patients or applying modalities ${ }^{11}$.

Based on the occupation and area of practice, Musculoskeletal and general physical therapy (which includes all) were having more prevalence than others, the reason could be treating more number of patients, less rest time, repeated activity. Also based on their post, more prevalence was found in both (academics and clinical), when compared it with academics or clinical alone. The possible explanation could be more work load of both clinical and academics which include treating patients and taking lecture that too in standing or sitting, which results into altered posture and resulting into WRMSD. This also accounts for total hours of working and sitting hours. More the hours of working results into more tiredness, fatigue less rest. In case of sitting hours results into more of back, upper back and neck pain and thus resulting into WRMSD 9 .

The most affected area was neck, then in lower back and hen was upper back. The reasons for that would be respondents who deals with excessive numbers of daily patients, lifting or transferring dependent patients and continuing to work while injured or hurt. No a day's therapists are working more on the devices like phone or laptop, which will keep the neck in a constant static position for a long time resulting into common neck disorders ${ }^{(2,14)}$.

The second objective of the study was to compare the association of WRMSD to Mental health status and Quality of life. There was a significant association with quality of life nut not with mental health. The reason for that was concluded that with pain, discomfort there was drastic change in quality of life, but not many effects on mental health status. The major factor that the participants were having affections was in environmental domain and physical domain in WHO-BREF. So it has concluded that WRMSD might have directly affected these domains rather than the others ${ }^{10}$.

Also in case of mental health status, some question like not able to concentrate, enjoying life, making decisions, participations, all these might have an affections on body, but the therapists might answers in as normal, as it would be a part of his/her work or adopted some coping strategies to overcome it.

\section{CONCLUSION}

Work-related musculoskeletal disorders are an important health risk within the physiotherapy profession. The prevalence of work-related musculoskeletal disorders among the Physiotherapists is $82 \%$. It is also concluded that the low back and neck are the most common sites susceptible to injury followed by upper back and thighs. Therefore, it is recommended that education programs on prevention and coping strategies for musculoskeletal symptoms in Physiotherapists should be made mandatory in order to decrease the risk of WRMSDs which will ultimately improve the quality of life.

\section{Limitations}

- As for prevalence study a larger sample size should be included.

- Also the type of chairs, table, computer use, the chair table arrangement should also been included in the assessment.

- Any pre-existing condition of the therapists was not included in the study.

\section{Future Recommendations}

1. As for prevalence study a larger sample size must be taken.

2. In detail analysis of musculoskeletal disorders must be taken, like in areas, neck pain, which conditions, the disability score, range of motion, strength of muscles.

\section{Acknowledgement: None}




\section{Conflict of Interest: None}

\section{Source of Funding: None}

\section{Ethical Approval: Approved}

\section{REFERENCES}

1. Buddhadev NP. Work-related musculoskeletal disorders: a survey of physiotherapists in Saurashtra region. Age (years). 2012;21(25):27-5.

2. Salleh NF, Sukadarin EH, Zakaria J. Preliminary study of musculoskeletal complaints and ergonomic risk factors among catering workers. Asia Pacific Environmental and Occupational Health Journal. 2017 Aug 18;3(1).

3. Gebreyesus $T$, Nigussie $K$, Gashaw $M$, Janakiraman B. The prevalence and risk factors of work-related musculoskeletal disorders among adults in Ethiopia: a study protocol for extending a systematic review with meta-analysis of observational studies. Systematic reviews. 2020 Dec;9(1):1-6.

4. Bae YH, Min KS. Associations between work-related musculoskeletal disorders, quality of life, and workplace stress in physical therapists. Industrial health. 2016 Jul 31;54(4):347-53.

5. Crawford JO. The Nordic musculoskeletal questionnaire. Occupational medicine. 2007 Jun 1;57(4):300-1.

6. Montazeri A, Harirchi AM, Shariati M, Garmaroudi G, Ebadi M, Fateh A. The 12item General Health Questionnaire (GHQ12): translation and validation study of the Iranian version. Health and quality of life outcomes. 2003 Dec;1(1):1-4.
7. Thadathil SE, Jose $\mathrm{R}$, Varghese $\mathrm{S}$. Assessment of domain wise quality of life among elderly population using WHOBREF scale and its determinants in a rural setting of Kerala. International Journal of Current Medical and Applied Sciences. 2015 Jun;7(1):43-6.

8. Maheshwari P, Soni R, Parkash N. Work Related Musculoskeletal Disorders: A Survey of Physiotherapists in Tricity. International Journal of Physiotherapy. 2015 Dec 1;2(6):1091-6.

9. Malarvizhi D, Kumar MK, Sivakumar VP. Prevalence of Work-Related Musculoskeletal Disorders among Clinical and Teaching Physiotherapists-An Observational Study. International Journal of Physiotherapy. 2017 Apr 1;4(2):86-92.

10. Bae YH, Min KS. Associations between work-related musculoskeletal disorders, quality of life, and workplace stress in physical therapists. Industrial health. 2016 Jul 31;54(4):347-53.

11. Wærsted M, Hanvold TN, Veiersted KB. Computer work and musculoskeletal disorders of the neck and upper extremity: a systematic review. BMC musculoskeletal disorders. 2010 Dec; 11(1):1-5.

How to cite this article: Sai Iyer, Labdhi Shah, Chitra Sharma. Prevalence of musculoskeletal disorders and its association with mental health and quality of life among working physiotherapists. International Journal of Science \& Healthcare Research. 2022; 7(1): 140-147. DOI: https://doi.org/10.52403/ijshr. 20220124 